\title{
Improved Data Dissemination using Intelligent Stimulus Mechanisms in IoT Network
}

\author{
${ }^{1}$ R.Radhika, ${ }^{2}$ Dr.K.Kulothungan \\ ${ }^{1,2}$ Department of Information of Science and Technology, College of Engineering Guindy, \\ Anna University, Chennai, Tamilnadu 600025, India, \\ rr1.techno@gmail.com
}

\begin{abstract}
Internet of Things (IoT) is virtually connecting object in the physical world to the internet, where a huge number of embedded devices relays on communication service offered by the internet protocols and these devices are termed as smart objects. Since the smart devices have low configuration it has constrained communication. During the communication, the data has to be disseminated with reliability. The enormous amount of data generated by heterogeneous devices do not achieve higher delivery ratio. Hence, reliable sensor data collection and appropriately disseminating data to targeted IoT device is critical in IoT environment. In the existing work, selfish behavior of each devices and the packet delivery delay are not considered. By observing this, the core challenge to disseminate the data efficiently under a unique network setting with multiple interest's data types. Two data dissemination schemes, namely the rule based data pull scheme and rule based data push scheme are proposed in this paper. The data pull scheme in IoT devices pull, the data from the data provider device, and in data push scheme the data providers generate data and push them to the intended users. In pull scheme, an effective mechanism known as cooperative game using intelligent agents is implemented. In addition, data sharing plays a crucial role between the devices where cooperativeness has been ensured in this work by cooperative game using intelligent agents and Pareto optimality. In data pushing model, a checking process is done using rules to monitor the receiver with best credits that gets the disseminated data form server which is processed by rule based online auction algorithm when comparing the Cooperative Scheme for Data Dissemination (CSDD) with existing model, this proposed model shows better performance in terms of data delivery ratio and delay in delivering the data to the destination.
\end{abstract}

\section{INTRODUCTION}

Internet of Things popularly reaches all users and is experiencing unpredictable growth. The transmission of enormous data generated by smart devices often relay on communication infrastructure, Internet connection acts as a back bone to achieve this smart infrastructure. An autonomous Internet of Things (IoT) network does not depend on any infrastructure but it exploits devices. It communicates with heterogeneous devices via short range signals or Device to Device (D2D) communication and Machine to Machine communication (M2M). An efficient data transfer can upgrade network performance and reduce communication cost. The transmission and the connection between the devices intermittent must be delay tolerant. The visual search for Internet of Things had profound impact leading to a great opportunity for smart devices among all the smart devices applications for gathering data in large scale, which may span ver several days [1]. Data transmission using effective scheduling algorithm increases the data collection under the constraints of radio link [2]. By ensuring the increase in data reception rate, it is possible to decrease packet loss with varying data rate and different network scale. The devices here are connected together to create, gather and share information which involves a series of transformation of data.

Therefore, the data delivery via IoT networks may be the last resort not only for its simplicity, greater delivery ratio and less delay but also for its effectiveness and optimistic delivery of data to the device through the server. The existing systems suffer from severe performance degradation especially due to heterogeneity of devices. The IoT network disseminates the data based on store, carry and forward. By considering this, the data pull and data push schemes are constructed. The transmission of data from a source to destination depends on the nodes that impact the data dissemination. The node participating in the dissemination process must be an interested node but which is not always be the case since selfish nodes also present. The selfish nodes decide not to help the other nodes to transmit the intended data and so such nodes have to be detected in order to avoid failure delivery.

A conditional statement that can be used to perform prediction by identifying the next data receiver is termed as a rule. Here the accuracy of a prediction is the only thing that really matters. The ability to take the decisions is crucial and have these rules stored in the form of IF.. THEN statement for identifying the efficient node to receive the data. Moreover intelligent agents are used to make accurate and collaborative decisions. The intelligent agents are classified into four type's namely simple reflex agents, multi agents, mobile agents and ant based intelligent agents. Here, the multi agent is employed in order to attain inherently intelligent solutions on data transfer. The agent could perform different and possibly independent tasks, but at the same time they cooperate during communication, in order to guarantee cooperativeness between the nodes. Cooperation among nodes are determined by the agents through node tightness and set of rules according to which all the agents are supposed to plan their actions. By 
the path of the monitoring process, each agent is able to establish whether its neighbors are cooperative. The major advantage of this agent is the scalability and reliable communication.

In this work, these problems are addressed by proposing novel data pull and data push schemes. These proposed schemes enhance the data dissemination by using a cooperative game theory and rule based online auction over the IoT network. It detects the selfishness of the nodes based on the credit and reward points. Hence, the best receiver is decided to enhance the performance and thus increasing the network behavior in terms of data dissemination.

The rest of the paper is organized as follows, in section II, Previous Work are reviewed and the Problem Statement is described. In section III, the details of the Proposed Work are presented. In section IV, Performance Evaluation is presented using the experimental results and comparisons. Finally, the work is concluded in section V.

\section{RELATED WORK}

Exchanging information data depends on the ability of the device to route data to other devices and disseminate data to other heterogeneous IoT network. Hence different types of communication may exist within the IoT. Data is disseminated between device to human and vice versa and device to distributed storage. In [3], communication could be single hop or multi hop. Single hop communication takes place via network infrastructure and in multi hop communication, devices depend on information for end to end communication between source and destination device. The authors classifies communication into inter domain and intra domain communications. Devices may communicate with storage agent to pass the captured data, update the stored data, or retrieve the stored data in order to make decision.

According to [4], the first and foremost requirement of the IoT is to provide the required connectivity between the devices. This provides effective communication in IoT device with performance in terms of access rate and the achieved network throughput gain. The coordinated interference and embedded automatic pairing are enhanced and compared to short range communication technologies for IoT device transmissions. Resource allocation is also an unaddressed problem in IoT devices with multiple cellular equipped environment. In [5], Data sharing protocol is used to ensure the data transmission. The data is signed by the data provider and is resigned by the transmitter to avoid repudiation in data sharing by doing so it assures the resource availability. In order to ensure the resource limitation of end user communication [6], the client need to participate in social trust group or in social reciprocity group leading to the key challenge of dividing the client into multiple social reciprocities and it is achieved by coalition game framework. In [7], the efficiency of the resource is improved by cooperative communication using coalition game theory. This uses merge and split solutions perusing better pay off for the user. The standard Routing Protocol for Low power and Lossy networks (RPL) doesn't provide high throughput [8] and so it suffers from severe congestion and packet loss which the limits in IoT applications. The Backpressure RPL acts an enhanced version of RPL to increase high throughput and adaptability. In [20], the fault tolerant routing protocol is considered to overcome the route failure in nodes, while disseminating the data form source to destination, this has the capability of reporting the error during data transmission and it mainly depends on the number of forward neighbour. The proactive protocol in [22], notifies the sender about the failure delivery of the data packet, the node id is stored by the neighbour node which receives the error message, using the query response message to check the neighbour in a round trip time to receive the data without failure.

The communication process in D2D is for exchanging information or forwarding data, the device must have the ability to route the data for other devices vice versa in the heterogeneous IoT network [3] irrespective of its underlying technology. The devices increase the volume of network traffic by generating data which affects the reliability, connectivity and communication speed. The effective solution addressed by the author is intelligent routing. To ensure the relay traffic between the devices, cooperativeness of the devices is necessary. In [9], code dissemination heterogeneity of IoT environment is considered to identify fake dissemination in advertisements in a colluding group, therefore it falls with issues like increased overhead and delay in dissemination. The feedback suppression and chaining technique [10] are used to handle feedback implosion and long tail problem. Here, the time threshold is monitored until the last propagation of the packet until then the nodes wait to send their feedback.

In Internet of Things data dissemination is first addressed in ADMin[11], it is a low-cost IoT framework that reduces the device energy consumption and the volume of data disseminated across the network. Based on IoT device disseminate data by monitoring streams based on run time knowledge. Interested node enhances the efficiency of data dissemination. In [12], a methodology is presented which is used to evaluate the use of different kinds of network channels and various data formats and maximize their cost effectiveness, and also it maps the data exchange volumes to the cost of data transfer usage. In [13], a utility based data computing in IoT is proposed for cooperatively collect data with the optimal price to obtain the profits during data sensing. GSD is presented in [14], it can disseminate information with huge delivery ratio, lowest overhead, least hop count and latency. Service based data forwarding scheme proposed in [15] provides altruism to forward the packets and the contribution of the relay node .Node with higher altruism is the preferred candidate for packet relaying. The altruism of 
node is low thus the effectiveness of incentive scheme is analyzed. In [16], proposed efficient data collection and dissemination mechanism decreases the amount of data transmission in order to increase the lifetime in IoT with Wireless Sensor Network.

The three phase approach [17] in device to device data dissemination handles altruistic and selfish behaviors of users are handled by message selection and cooperation pairing which accommodate the incentive constraints. A survey on data dissemination delay tolerant network is presented in [18]. This work analyses the features of delay tolerant network and its taxonomies. They also studied the communication correlation between the message replication and individual or group by considering the social and opportunistic based message forwarding mechanisms. In [19], the content distribution problem is addressed in Device to Device multi hop communication by considering cross layer optimization involving frequency, time and space the network where the delay is reduced. The coalition game based payoffs is formulated to disseminate the content in minimal time duration. A multicast communication approach is proposed [21] which provides efficient and secure reprogramming protocol. For heterogeneous IoT applications, the challenges are over come to provide optimization for the constrained nodes to reduce the communication and computational overhead. The reliability aware intelligent data dissemination is proposed [23], it handles broadcast storm problem. In [24], Honey Bee optimization is used to find the optimal route to deliver the data with low cost, it extends the network life time in terms of energy efficiency and scalability. The agents of things in IoT [25] interact with others by software agent this act as a reasoning agent to upgrade the intelligence in the devices by updating the software's. In Agent based IoT devices in Intelligent Home Security System [26] acquired information through agents, the agent covers various standards and protocols to cooperate with each other to coordinate multiple task.

In this section, an incentive scheme for data pull model and data push to stimulate the cooperation among nodes is proposed to find the best strategy for each selfish node.The IoT devices in such a network can be either selfish or cooperative. If the node is cooperative then it disseminates data voluntarily. The selfish node it is reluctant for other nodes energy and bandwidth. It also neglects to carry any messages other than the ones interested by itself by doing so at one point of time data's are not shared among the nodes, it leads to poor network performance. In this proposed work, the coherent behavior of selfish node is considered. The selfish node disseminate data only when it gains benefit. The credit based scheme is used to promote participation of the selfish node. Credit points are rewarded only when the final deliverer delivers the data to the end user. While disseminating the data more copies of data is created. The first copy of the data is
TABLE I

DEFINITION OF NOTATIONS

\begin{tabular}{ll}
\hline \hline Notation & \multicolumn{1}{c}{ Definition } \\
\hline $\boldsymbol{\tau}_{\mathrm{n}}(\mathrm{i})$ & Direct tightness of Node $\mathrm{n}$ in Interest i \\
$\delta_{\mathrm{n}}(\mathrm{i})$ & InDirect tightness of Node $\mathrm{n}$ in Interest i \\
$\boldsymbol{\chi}_{\mathrm{n}}(\mathbf{i})$ & Overall tightness of Node $\mathrm{n}$ in Interest $\mathrm{i}$ \\
$\mathrm{P}_{\mathrm{n}} \mathrm{d}(\mathrm{i})$ & expected credit reward \\
$\mathrm{M}^{\mathrm{p}}(\mathrm{i})$ & potential value \\
$\left(\mathbf{Y}_{\mathrm{n}}, \mathbf{Y}_{\mathbf{k}}\right)$ & utility gains of Nodes $\mathrm{n}$ and $\mathrm{k}$ \\
$\mathrm{E}^{\mathrm{p}}(\mathrm{i})$ & Estimated number of copies of Message \\
$\left(\mathrm{Q}_{\mathrm{n}}, \mathrm{Q}_{\mathrm{k}}\right)$ & Status quo point \\
$\mathrm{ADF}$ & Arrival Distribution Function \\
$\Phi$ & Observed receiver's time. \\
$\mathrm{ITA}$ & Density of Independent T time Arrival \\
$\mathrm{WT}$ & Waiting time \\
Notation & Definition \\
\hline \hline
\end{tabular}

paid by the receiver. A cooperative game is used between two nodes for a cooperative communication and a game theory is applied to reach optimal solution.

\section{PROPOSED WORK}

Since selfish behavior of the device reduces the performance of routing many incentive mechanisms have been studied to stimulate individual selfish device to be cooperative, the incentive strategies are classified into two strategies that is reputation based and credit based approach Data Routers are responsible for transferring the data. A novel layer concatenation technique to with stand abnormal action of selfish nodes is adapted [23]. The detailed definition of notations are given in a Table I. The credit based schemes form of credit introduced or regulated the packet forwarding relationships among different devices a virtual currency is used. Credit points are used to obtain forwarding service from any device in the network, each forwarding request, an extra amount is charged by the virtual bank to the sender and the intermediate device redeem their rewards at the bank after successful delivery. A copy adjustable incentive scheme [24] is a reputation based incentive scheme where the credit concept is virtual to simulate the selfish to forward data. It detects the misbehavior of the nodes and remove them from the network.

The data is forwarded based on their reputation of the node. Nodes with good reputation can receive service form other nodes. On the other hand, nodes with bad reputations will be denied participation in network, thus it will help the nodes motivated to cooperate. In reputation-based schemes, forwarding services are provided to nodes depending on their reputation. When a node provides services for other nodes, it gets good reputation. Nodes with good reputations can receive services from other nodes. On the contrary, miss behaving nodes get bad reputations and will be denied participation in the network. The selfish node is detected and the punishment motivates nodes to cooperate. IoT devices in the real world 
can be cooperative or selfish. Generously it carries and shares data for others. In other way, a device with selfishness is used to maximize its own benefits only to reward its own behavior. IoT device with selfishness and rational behavior is taken into account where a device is driven by its interest's. Data is disseminated only if it gains benefit.

The incentive of a message under an IoT network with multiple interest's data types is based on the rewards obtained by the nodes. The credit points or rewards relies on successful transmission of the data to the end user. Thus the value of a message for an intermediate node highly depends on its probability to deliver the message. Such probability itself is nontrivial to estimate. Moreover, a message is usually desired by multiple users. Therefore, it can be potentially vend multiple times to different receivers. The problem is further complicated by message duplication that is common in data dissemination in IoT network, where multiple copies of a message may be delivered to the same receiver but only the first copy is paid. While the above properties apply to both data pull and push there exists a fundamental difference between them. In data pull model, a receiver intends to receive and consume data, and thus, should pay for the final deliveries; while in data push model, a data provider intends to disseminate its personalized data, and thus, is deemed as the payer. However, it does not know how many nodes will involve in packet delivery and which node it should pay for. These characteristics together make the development of incentive mechanisms a unique, interesting, and challenging problem.

\section{A. Cooperative Scheme for Efficient Data Dissemination}

The tightness indicates how often a node delivers data in an interest type to its $\operatorname{sink}(\mathrm{s})$. Its value intrinsically depends on disseminating data, and aggregates direct and indirect contacts. The former, i.e., the direct tightness of Node $\mathrm{n}$ in Interest $\mathrm{i}$, indicates how often Node $\mathrm{n}$ directly meets a node that is the sink of Interest i. The latter is the indirect tightness, which captures how often Node $\mathrm{n}$ delivers data to the sinks of Interest $i$ via other nodes indirectly. Here alternating direction method of multipliers are used since they are the most effective schemes for tighter bounds, This bound is much preferred in large scale network data, the quantization resolution, and the number of user is estimated and employed in many prior works, to maintain and update the node tightness [25].

More specifically, each node maintains a timer. If there is no contact with other nodes within an interval, the timer expires, generating a timeout event. The knowledge about the term are as follows.

1. Interest acts as a type of data that a node wishes to disseminate

2. Source of an interest is the data generated by the node to match the interests.

3. The sink node is the consumed data that matches the corresponding interest. A node may act as a sink or sources for multiple interest.

4. Tightness of node is represented as a node can contact the sinks of interest directly or indirectly within a time interval. When the time elapses the timeout is generated

The Direct Tightness and Indirect Tightness's manipulated as follows:

\section{Direct Tightness}

1. Let $\boldsymbol{\tau}_{\mathrm{n}}$ (i) - Direct tightness of Node $\mathrm{n}$ in Interest $\mathrm{i}$

2. $\boldsymbol{\tau}_{\mathrm{n}}(\mathrm{i})=0$, and updated at every contact with the node matching Interest $i$ or a timeout event.

3. The function is updated the Direct tightness is computed for contact in equation (1) and for time out equation (2)

$$
\tau_{\mathrm{n}}(\mathrm{i})=(1-\lambda) * \tau_{\mathrm{n}}(\mathrm{i})+\lambda
$$

For time out $=(1-\lambda) * \tau_{\mathrm{n}}(\mathrm{i})$

A constant $0 \leq \lambda \leq 1$ is employed to keep partial memory of previous status.

\section{Indirect Tightness}

1. Let $\delta_{\mathrm{n}}(\mathrm{i})$ - Indirect tightness of Node $\mathrm{n}$ in Interest $\mathrm{i}$.

2. The function is updated the Indirect tightness is computed for contact in equation (3) and for time out equation (4)

$$
\delta_{\mathrm{n}}(\mathrm{i})=(1-\mu) * \delta_{\mathrm{n}}(\mathrm{i})+\mu \tau \mathrm{k}(\mathrm{i})
$$

For Time out $=(1-\mu) * \delta_{\mathrm{n}}(\mathrm{i})$

Where $\left.\boldsymbol{\tau}_{\mathrm{n}}(\mathbf{i})\right)$ and $\boldsymbol{\delta}_{\mathrm{n}}(\mathbf{i})$ are the direct and indirect tightness of Node $\mathrm{n}$ in Interest $\mathrm{i}$. We adopted the exponentially weighted moving average (EWMA) [11], which is one of the most effective schemes for online estimation and has been employed in many prior works, to maintain and update the nodal tightness.

More specifically, each node maintains a timer. If there is no link with other nodes within an interval of the timer expires, generating a timeout event. Let $\boldsymbol{\tau}_{\mathrm{n}}$ (i) denotes the direct tightness of node $\mathrm{n}$ in Interest i. $\boldsymbol{\tau}_{\mathrm{n}}$ (i) is initialized to zero, and updated at every contact with the node matching Interest $i$ or a timeout event.

Where $0 \leq \mu \leq 1$ is the previous status and $\tau_{\mathrm{k}}$ (i) is the direct tightness to any node with Interest $i$ by node. The indirect tightness is considered here since a node only receives rewards through direct delivery. The reason is that indirect tightness can help nodes to trade valuable messages.

By assuming node A owns message $\mathrm{m}$ in Interest $\mathrm{i}$, but has a low direct tightness to any receiver in Interest i. At the same time, node $\mathrm{B}$ has a high direct tightness to receivers in Interest $\mathrm{i}$. When node A and B meet, message $\mathrm{m}$ can be a very competitive message for node A to trade with node B. Thus, the indirect tightness also contributes to data delivery and rewarding process assuming indirect contacts involve two hop relaying only in the following discussions. According to the direct and indirect tightness $\chi_{n}(\mathrm{i})$ is denoted as the overall tightness of node $\mathrm{n}$ in Interest $\mathrm{i}$.

$$
\chi_{\mathrm{n}}(\mathrm{i})=1-(1-\tau \mathrm{n}(\mathrm{i}))(1-\delta \mathrm{n}(\mathrm{i}))
$$

\section{B. Data Pull Scheme}

The incorporation of data dissemination with selfish nodes and multiple interest data types is a challenging tasks. The 
proposed work focuses on disseminating data messages to corresponding sinks with both delay and traffic overhead as low as possible. The two data dissemination models have been proposed from two different perspectives: the data pull model where IoT node receive data from data providers, and the data push model where data providers generate personalized data and push the data to the intended users.

This effective mechanisms is to estimate the expected credit reward of a data that helps intermediate nodes to evaluate the potential reward of a message. Nodal message communication is formulated as a two node cooperative game. The data pull model eliminates the needs of accurate knowledge about the credits that data providers should pay. The check bidding process is formulated as an auction model to further accelerate the circulation of credits network.

Due to frequent partitioning and lack of an end-to-end path, it is impractical for a node to manage reputation of its neighbors. It is also difficult to estimate the number of intermediate nodes that would participate in packet forwarding. In order to set a proper initial credit for the packet, as required in the credit-based systems this scheme provides facilities the data pull model, where a receiver intends to receive and consume data, and thus, should pay for the final deliveries, the data push model, where a data provider intends to disseminate its personalized data, and thus, is deemed as the payer. Selfishness has been investigated in the context of IoT.

\section{Generation of Credit Points}

Data providers generate the unique ID and the value of virtual check $\lambda_{p}$ in packet $p$ is the amount of virtual credits. Each intended receiver receives the data packet and the data provider will pay them. The data provider has the power to decide the value of the virtual check. The higher the value the stronger the interest often results in faster dissemination of the data. The virtual check has to be signed by the receiver or it is void. The deliverer is rewarded with the sign by the receiver.

Optimal Deliveries is achieved when the maximum number of receivers receives the data sent by the maximum deliveries $\varepsilon_{\mathrm{p}}$ of Packet $\mathrm{p}=\lambda_{\mathrm{p}} \times \varepsilon_{\mathrm{p}}$ the credits provided by the provider would like to pay for disseminating the data. Moreover it relays on the number of users in the network who are interested in the data packet. The data provider can initialize $\varepsilon_{\mathrm{p}}$ using counting algorithm and it is updating during dissemination. The value of data packet depends on the following factors:

1. Node with higher probability to meet the receivers and to earn signed checks.

2. Node with higher deliveries to obtain multiple signed checks. It relates itself and remains same for all nodes.

3. The check received node will get the appraisal based on the probability of being cashed.

If there is non cooperativeness exits among nodes the interested messages are disseminated from data providers. In the cooperative scheme, the nodes are fully cooperative and always choose the most valuable message to carry after satisfying its own interests.

Credit and rewards are employed to facilitate a node to determine when it should trade.

1. Let $P_{n} d(i)$ - expected credit reward it depends on appraisal

2. $\mathrm{M}^{\mathrm{P}}(\mathrm{i})$ - potential value, Potential value depends on two factors

i) Node $n$ gains credit points when it delivers data to sink Pnd(i).

ii) Sink is interested in one copy and thus pays for the first received.

3. After completing the tradeoff multiple copies of the same data is present in each node, this leads to lower the probability of transmitting a copy to a sink, thus we have

$$
P_{n} d(i)=M^{p}(i) \times \varphi_{n}(i) / E^{p}(i)
$$

\section{Cooperative Game Theory}

The two nodes in the game act as the players and are rational and selfish, depending on whether it brings benefit or harm to the players. A node can usually improve its function by obtaining a new data. The two-person cooperative game allows players to reach a binding agreement, based on their possibly conflicting interests. The non-cooperative game that doesn't allows the non-binding agreement between players. By ensuring benefits of the players it has to reach a binding agreement. The two person cooperative game for obtaining optimal solution is maximized by

$$
\left(Y_{n}, Y_{k}\right)=\arg \max \left(Y_{n}-Q_{n}\right) \times\left(Y_{k}-Q_{k}\right)
$$

Where $\left(\mathrm{Q}_{\mathrm{n}}, \mathrm{Q}_{\mathrm{k}}\right)$ - Status quo point. $\mathrm{Y}_{\mathrm{n}}, \mathrm{Y}_{\mathrm{k}}$ is set to $(0,0)$ is used to obtain a feasible solution.

The data dissemination is determined by $Y_{\mathbf{n}}$ (or $Y_{k}$ ) Therefore, the optimal solution yields an optimal set of data that should be exchanged between Nodes $\mathrm{n}$ and $\mathrm{k}$ respectively.

Each node owns a large number of data and it takes exponential time to identify. This work considers one set of data at a time $d_{n} \in m_{n}$ and $d_{k} \in m_{k}$, where $d_{n}$ and $d_{k}$ are exchanged between nodes $\mathrm{n}$ and $\mathrm{k}$. The process is repeated until nodes $\mathrm{n}$ and $\mathrm{k}$ lose their connection and the intermediate queue is empty. The correct decision is made based on the pay off or utility, it's impractical to obtain perfect rationality this is due to intractability. In data pull model, it is the responsibilities of the receiver to pay, since they are wishing to get their own interested data form the deliverers.

However in data push model, it is the data providers who wish to disseminate their personalized data to the intended user are intended to pay for delivery service. The deliverer gets the credit point from the data provider who generated that data. In IoT networks, the data provider can personalized data 
and pull the data to the intended users. During data dissemination, the signed checks and data packets can be traded between the two nodes. It is crucial to decide which data packet or signed check should be transmitted. A node tries to maximize its own benefit, which affects the other nodes interest due to the node selfishness. Trading is done when a node needs a data packet or signed check form the other node. Conflicts arise during the packet exchange and it must not credit one node and degrade the other. During the disseminating process, the cooperativeness has to be ensured with the game theory. The key challenge encounted during node delivery is to identify which node helps to deliver the data and it also finds the deliverer getting the credit timely and it is handled by data pull model.

\section{E. Data push Scheme}

In order to maximize the receiver benefits, a formulation of online auction algorithm is used in order to satisfy both sender and receiver. In order to maximize the receiver benefits, a formulation of online auction algorithm is used in order to satisfy both sender and receiver. Three promising criteria associated with the check once it is added into the buffer are listed below.

1. Upper bound credit- It indicates the expected value the vendor wishes.

2. Lower bound credit - indicated as the minimal value that vendor wants

3. Listing period- This indicates how long the check to be debited.

Waiting time [0, WT] is considered in this model to observe all the receivers. The goal here is to maximize the probability of selecting the best receiver form unknown number of receivers. The upper bound and lower bound threshold values can be fixed based on the experimental results. The arrival distribution function is calculated using equation (8) as

$$
\operatorname{ADF}(\varphi)=\int_{0}^{\mathrm{t}} \mathrm{t}_{\mathrm{arr}}(\mathrm{s}) \mathrm{ds}, 0 \leq \mathrm{t} \leq \mathrm{WT}
$$

$t_{\text {arr }}$ is the density of independent time arrival and $\varphi$ is the observed receiver's time.

Selection process of best receiver based on online auction scheme is explained using the following algorithm. The steps involved are

Applying rule 1:

1. IF credit count is greater than the boundary credit then It has to write true.

2. Compute $\mathrm{T}$ which is the cut off time

3. $\operatorname{ADF}(\varphi)=(1 / \mathrm{e})$

4. Check if the current time less than cut of time

5. Check whether the credit count is greater than previous credit count.

6. Write if previous max count equal to credit count.

7. End all if conditions

Applying rule 2:

1. IF total of max credit and credit count is greater than debit count and also greater than low boundary credit

2. Write true else write false
By doing so, it solves the secretary problem with unknown number of receivers.

\section{Performance Evaluation}

The simulation has been carried out using Network Simulator-3 (NS-3) and analysis is shown in Table1.1. The evaluation and the performance are validated based on the effectiveness of proposed Cooperative Scheme for Data Dissemination (CSDD) through this simulation. The simulation environment, performance metrics and simulation results are presented in this section. A comparative study on the metrics, with existing protocol namely Geo-Social Distance-Based Data dissemination method (GSD), are also presented in the graphs below. The simulation is performed for the varying queue size and interest types. Table II indicates the simulation parameter and topology are considered.

\section{Simulation Parameter}

TABLE II

NETWORK PROPERTIES FOR SIMULATION

\begin{tabular}{|l|l|}
\hline Parameter & Value \\
\hline Simulator & NS-3.25 \\
\hline Topology & $\begin{array}{l}\text { Random IOT things placement ,one server and } \\
\text { three gateway }\end{array}$ \\
\hline Number of nodes & 100 \\
\hline Wifi Data Rate & 1 Kbits/s to 10 M bits/s \\
\hline Propagation Model & Log Distance Propagation Loss Model \\
\hline Physical Model & Yans wifi Phy model \\
\hline Channel Model & Yans wifi Channel Model \\
\hline Queue Size & 50 to 300 \\
\hline Traffic type & CBR,UDP \\
\hline $\begin{array}{l}\text { Number of interest } \\
\text { types }\end{array}$ & $5,10,15,20 \ldots, 30$ \\
\hline Routing Algorithm & CSDD \\
\hline Packet size & 1024 \\
\hline Mac protocol & $802.15 .4 \mathrm{e}$ \\
\hline
\end{tabular}

In this section, the experimental data and the performance results are presented, the performance evaluation results are narrated along with graphs.

The Packet Delivery Ratio (PDR) increases with increase in Queue size. CSDD is observed to be stable and the increase in PDR is very marginal with a variation of higher than $4 \%$. GSD has nearly $10 \%$ reduction and this is significant improvement by the proposed CSDD. Figure.1 depicts packet delivery ratio (PDR) for given Queue size involved in transmission. 


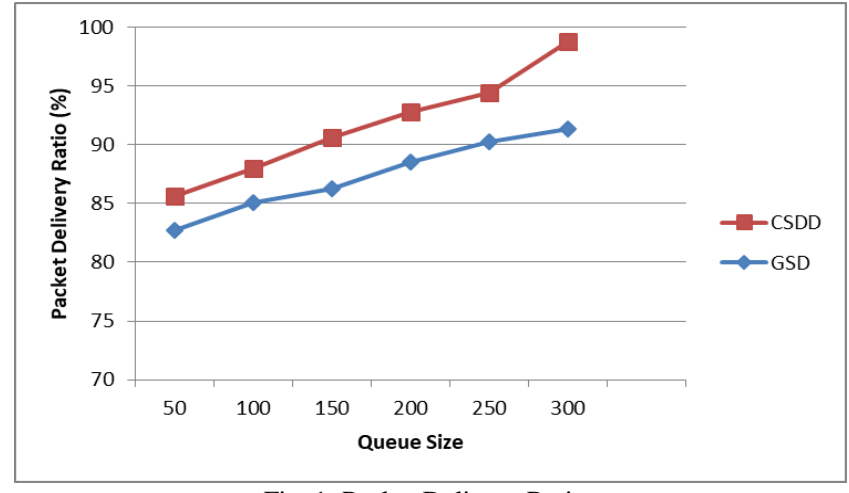

Fig. 1. Packet Delivery Ratio.

Fig. 2 shows the results obtained from Average of Selfishness Aware Routing and cooperative scheme for efficient data dissemination. It shows the average delay, defined as the time taken for transmitting the packet inside IOT network. From Figure 2, it is observed that the CSDD suffers the least delay compared to the GSD. In this case also, the proposed CSDD proves to be the best compared to existing GSD. The increase of queue size has less impact on the performance of the proposed CSDD scheme, because it exchanges messages based on interests of individual nodes, aiming to promote rewards. Thus the nodes do not aggressively utilize the additional queuing space.

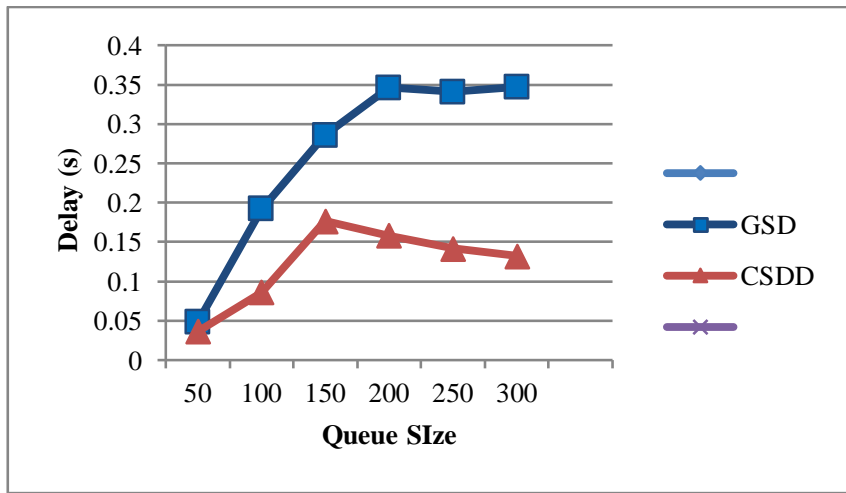

Fig. 2. Average Delay

Fig. 3 presents, the overhead with respect to the queue size. Here, the overhead increases with increase in the queue size. As the queue size increase, the number of transmissions also increase. CSDD has less overhead compared to GSD. CSDD has the optimum balance of both reliability shown by the PDR and efficiency achieved by reduced Routing overhead.

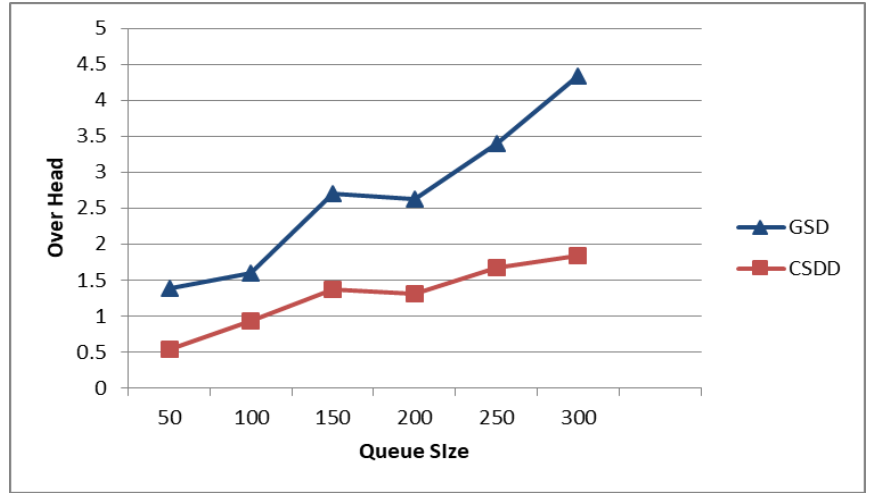

Fig. 3. Overhead.

Figure. 4 depicts the impact of the number of interest types in the network. From Fig. 4 it is observed that, with the increase of the number of interest types, the delivery rate decreases under Self Exchange, CooperRdm and Cooperative schemes, but increases slightly. This is because the cooperation between nodes in Incentive improves the possibility of delivering messages to each other when nodes have more interests.

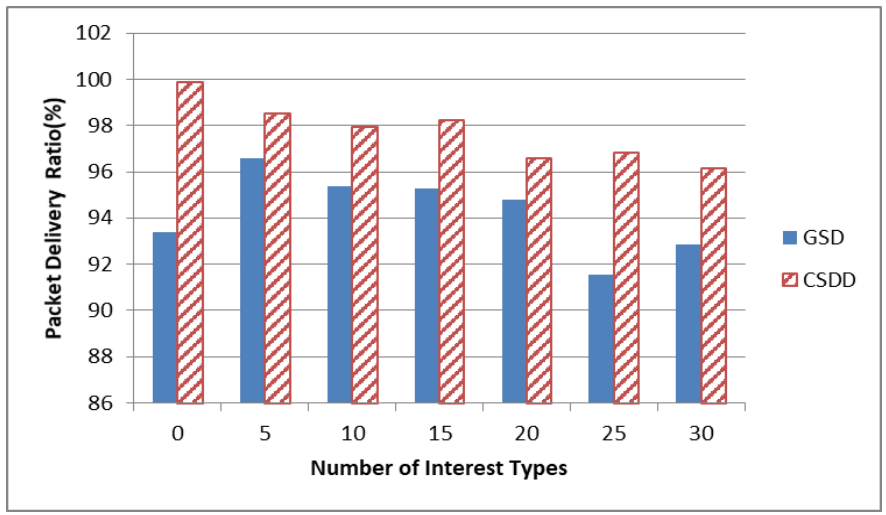

Fig.4. Packet Delivery Ratio

We observe in Figure. 5 that the average delay of all schemes slightly increase except the direct scheme.

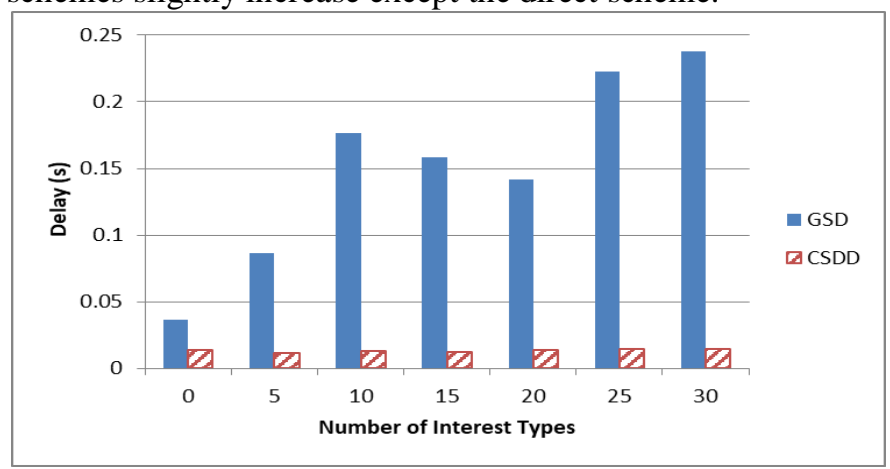

Fig. 5. Delay

From Fig. 6, we notice that the overhead of the proposed CSDD is stable. This is because a node only chooses the most beneficial messages to trade and never wastes its resources to free-riders. 


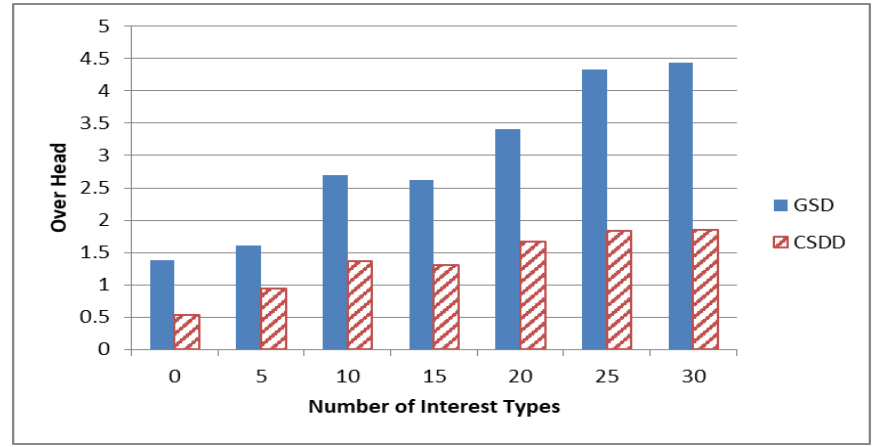

Figure 6: Overhead

Figure 6 shows the overhead analysis between GSD and CSDD.

\section{CONCLUSION}

In this work, we have addressed the problem of data dissemination in Internet of Things with selfish nodes in IoT, here there are two types of users which are the data provider and the device user. Here, instead of geo- distance based approach, we have proposed two model namely rule based Data push model and rule based Data pull model. In data pull model, we proposed reward and penalty based schemes which help the devices to evaluate their neighbor's behavior. For this, reward is ensured by cooperative game intelligent agent and pareto optimality approach. Penalty or Reward are given based on their behavior. The data push scheme used virtual checking process to identify about whom and how much data it's transmitting from server, this process is be done by using rule based online auction algorithm. Using this, both data push and pull model, we identified selfish behavior and we can achieve greater delivery ratio and less delay comparing to Geo-Social Distance-Based Data dissemination method.

\section{REFERENCES}

[1] Nunes, Luiz, Julio Estrella, Luis Nakamura, Rafael de Libardi, Carlos Ferreira, Liuri Jorge, Charith Perera, and Stephan Reiff-Marganiec. "A distributed sensor data search platform for internet of things environments." arXiv preprint arXiv:1606.07932 (2016).

[2] Chaturvedi, Tanmay, Kai Li, Chau Yuen, Abhishek Sharma, Linglong Dai, and Meng Zhang. "On the design of MAC protocol and transmission scheduling for Internet of Things." In Region 10 Conference (TENCON), 2016 IEEE, pp. 2000-2003. IEEE, 2016.

[3] Bello, Oladayo, and Sherali Zeadally. "Intelligent device-to-device communication in the internet of things." IEEE Systems Journal10, no. 3 (2016): 1172-1182.

[4] Elmesalawy, Mahmoud M. "D2D Communications for Enabling Internet of Things Underlaying LTE Cellular Networks." Journal of Wireless Networking and Communications 6, no. 1 (2016): 1-9.

[5] Zhang, Aiqing, Jianxin Chen, Rose Qingyang Hu, and Yi Qian. "SeDS: Secure data sharing strategy for D2D communication in LTE-Advanced networks." IEEE Transactions on Vehicular Technology 65, no. 4 (2016): 2659-2672.

[6] Cao, Yang, Tao Jiang, Xu Chen, and Junshan Zhang. "Social-aware video multicast based on device-to-device communications." IEEE Transactions on Mobile Computing 15, no. 6 (2016): 1528-1539.

[7] Díaz, Luis M. Gato, Meiby Ortiz Bouza, and Jorge Torres Gómez. "Modeling cooperative communications using game theory: applications for cognitive radios." Revista Científica de Ingeniería Electrónica, Automática y Comunicaciones 38, no. 2 (2017): 36-48.
[8] Tahir, Yad, Shusen Yang, and Julie A. McCann. "BRPL: Backpressure RPL for High-throughput and mobile IoTs." IEEE Transactions on Mobile Computing (2017).

[9] Kim, Jun Young, Wen Hu, Hossein Shafagh, and Sanjay Jha. "SEDA: Secure Over-The-Air Code Dissemination Protocol for the Internet of Things." IEEE Transactions on Dependable and Secure Computing (2016).

[10] Suzuki, Makoto, Chun-Hao Liao, Sotaro Ohara, Kyoichi Jinno, and Hiroyuki Morikawa. "Wireless-transparent sensing." In Proceedings of the 2017 International Conference on Embedded Wireless Systems and Networks, pp. 66-77. Junction Publishing, 2017.

[11] Trihinas, Demetris, George Pallis, and M. Dikaiakos. "ADMin: Adaptive Monitoring Dissemination for the Internet of Things." In IEEE INFOCOM. 2017.

[12] Martínez, Juan A., José L. Hernández-Ramos, Victoria Beltrán, Antonio Skarmeta, and Pedro M. Ruiz. "A user-centric Internet of Things platform to empower users for managing security and privacy concerns in the Internet of Energy." International Journal of Distributed Sensor Networks 13, no. 8 (2017): 1550147717727974.

[13] Paschou, Mersini, EvangelosSakkopoulos, EfrosiniSourla, and Athanasios Tsakalidis. "Health Internet of Things: Metrics and methods for efficient data transfer." Simulation Modelling Practice and Theory 34 (2013): 186-199.

[14] Hui, Yilong, Zhou Su, and Song Guo. "Utility based data computing scheme to provide sensing service in internet of things." IEEE Transactions on Emerging Topics in Computing (2017).

[15] Alduais, N. A. M., J. Abdullah, A. Jamil, and L. Audah. "An efficient data collection and dissemination for IOT based WSN." In Information Technology, Electronics and Mobile Communication Conference (IEMCON), 2016 IEEE 7th Annual, pp. 1-6. IEEE, 2016.

[16] Zhao, Yiming, Wei Song, and Zhu Han. "Social-aware data dissemination via device-to-device communications: Fusing social and mobile networks with incentive constraints." IEEE Transactions on Services Computing (2016).

[17] Sobin, C. C., VaskarRaychoudhury, Gustavo Marfia, and AnkitaSingla. "A survey of routing and data dissemination in delay tolerant networks." Journal of Network and Computer Applications67 (2016): 128-146.

[18] Kim, Jun Young, Wen Hu, Hossein Shafagh, and Sanjay Jha. "SEDA: Secure Over-The-Air Code Dissemination Protocol for the Internet of Things." IEEE Transactions on Dependable and Secure Computing (2016).

[19] Dua, Amit, Neeraj Kumar, and Seema Bawa. "ReIDD: reliability-aware intelligent data dissemination protocol for broadcast storm problem in vehicular ad hoc networks." Telecommunication Systems 64, no. 3 (2017): 439-458.

[20] Kulothungan, K., S. Ganapathy, S. Indra Gandhi, and P. Yogesh. "Intelligent secured fault tolerant routing in wireless sensor networks using clustering approach." International Journal of Soft Computing 6, no. 5 (2011): 210-215.

[21] Xu, Chen, Junhao Feng, Zhenyu Zhou, Jun Wu, and CharithPerera. "Cross-Layer Optimization for Cooperative Content Distribution in Multihop Device-to-Device Networks." IEEE Internet of Things Journal (2017).

[22] Kulothungan, K., J. Angel Arul Jothi, and A. Kannan. "An adaptive fault tolerant routing protocol with error reporting scheme for wireless sensor networks." European Journal of Scientific Research 16, no. 1 (2011): 1932.

[23] Ning, Zhaolong, Li Liu, Feng Xia, Behrouz Jedari, Ivan Lee, and Weishan Zhang. "CAIS: a copy adjustable incentive scheme in community-based socially aware networking." IEEE Transactions on Vehicular Technology 66, no. 4 (2017): 3406-3419.

[24] Selvi, M., C. Nandhini, K. Thangaramya, K. Kulothungan, and A. Kannan. "HBO based clustering and energy optimized routing algorithm for WSN." In Advanced Computing (ICoAC), 2016 Eighth International Conference on, pp. 89-92. IEEE, 2017.

[25] Chan, Tsung-Yen, Yi Ren, Yu-Chee Tseng, and Jyh-Cheng Chen. "eHint: An Efficient Protocol for Uploading Small-Size IoT Data." In Wireless Communications and Networking Conference (WCNC), 2017 IEEE, pp. 1-6. IEEE, 2017.

[26] Rivera, Diego, Luis Cruz-Piris, German Lopez-Civera, Enrique de la Hoz, and Ivan Marsa-Maestre."Applying an unified access control for 
IoT-based Intelligent Agent Systems." In Service-Oriented Computing and Applications (SOCA), 2015 IEEE 8th International Conference on, pp. 247-251. IEEE, 2015.

[27] Peng, Zhaoqing, Takumi Kato, Hideyuki Takahashi, and Tetsuo Kinoshita. "Intelligent home security system using agent-based IoT Devices." In Consumer Electronics (GCCE), 2015 IEEE 4th Global Conference on, pp. 313-314. IEEE, 2015. 